\title{
DESEMPENHO DE ALGORITMOS PARA CONTAGEM AUTOMÁTICA DE ÁRVORES JOVENS E ADULTAS A PARTIR DE DADOS VANT
}

Naiany Bonamichi Silva', Ana Paula Dalla Corte ${ }^{2}$, Luani Rosa de Oliveira Piva ${ }^{3}$, Ângela Maria Klein Hentz ${ }^{3,}$ Carlos Roberto Sanquetta ${ }^{2}$

${ }^{1}$ Acadêmica do Curso de Engenharia Florestal, Universidade Federal do Paraná,

Curitiba, Brasil.E-mail: naianybonamichii@gmail.com

${ }^{2}$ Professor (a) Doutor (a) do Departamento de Ciências Florestais, Universidade

Federal do Paraná, Curitiba, Brasil

${ }^{3}$ Doutoranda no Programa de Pós-Graduação em Engenharia Florestal, Universidade Federal do Paraná, Curitiba, Brasil

Recebido em: 22/09/2018 - Aprovado em: 23/11/2018 - Publicado em: 03/12/2018 DOI: 10.18677/EnciBio_2018B31

Esse trabalho objetivou testar o desempenho do software LiDAR360 e da ferramenta TreeDetect para detecção e contagem automática de árvores adultas e jovens, através de produtos gerados a partir de imagens VANT. A área de estudo, que localiza-se em Telêmaco Borba - PR, consiste de plantios jovens de Eucalyptus grandis $\mathrm{x}$ Eucalyptus urophylla e plantios adultos de Eucalyptus spp., com espaçamento de $3,75 \times 2,4 \mathrm{~m}$. Utilizaram-se 20 fotografias aéreas coletadas em setembro de 2015 com VANT eBee-Ag equipado com câmera RGB Canon S110. Realizou-se o pré-processamento no software Agisoft PhotoScan Professional, visando a geração do ortomosaico e nuvem de pontos. Primeiro avaliou-se a eficiência dos algoritmos em plantios jovens: LIDAR360 (Tj1) e TreeDetect (Tj2). Num segundo momento avaliou-se em plantios adultos: LIDAR360 (Ta1) e TreeDetect (Ta2). Para comparação, usou-se a testemunha jovem (Tj0) e a testemunha adulta ( $\mathrm{Ta} 0)$, ambos resultantes de contagem em tela. Considerou-se na análise três parâmetros: (a) indivíduos corretamente identificados, (b) erros de omissão e (c) erros de comissão. Para verificar a ocorrência de diferença estatística entre os tratamentos com relação às testemunhas, aplicou-se o teste t pareado, a um nível de $5 \%$ de probabilidade. Observou-se a identificação de $25,83 \%$ em Tj1 e $104,54 \%$ em Tj2 em relação a Tj0. Nos adultos a identificação foi de $68,44 \%$ em Ta1 e de $119,58 \%$ em Ta2, em relação a Ta0. Em todos os casos, os tratamentos não diferiram estatisticamente das testemunhas. Recomenda-se testar os algoritmos em outras áreas e em fotografias com melhor qualidade.

PALAVRAS-CHAVE: automação de inventário, nuvem de pontos, ortomosaico.

\section{PERFORMANCE OF ALGORITHMS FOR AUTOMATIC COUNT OF YOUNG AND ADULT TREES FROM UAV DATA}

\section{ABSTRACT}

This study aimed to test the performance of LiDAR360 software and TreeDetect toolbox to detect and automatic count the total number of young and adult trees, based in products from UAV imagery. The study area is located in Telêmaco BorbaPR and presents young plantation of Eucalyptus grandis $\times$ Eucalyptus urophylla and 
adult plantation of Eucalyptus spp., with plant spacing of $3.75 \times 2.4 \mathrm{~m}$. Twenty aerial photographs collected in September of 2015 with an eBee-Ag UAV, equipped with camera RGB Canon S110, were used. The pre-processing was done with Agisoft PhotoScan Professional edition software, aiming the generation of an orthomosaic and point clouds. The efficiency of the algorithms was evaluated first in young plantation: LiDAR360 (Tj1) and TreeDetect (Tj2), and in a second moment it was evaluated in adult plantations: LiDAR360 (Ta1) and TreeDetect (Ta2). Young (Tj0) and adult $(\mathrm{TaO})$ controls, both resulted from manual count in screen, were used for comparison. Three parameters were analyzed: (a) correctly identified individuals, (b) omission errors and (c) commission errors. The t paired test, with $5 \%$ level of probability, was used to check the statistical difference between the treatments and controls. The number of identified trees was $25.83 \%$ in $\mathrm{Tj} 1$ and $104.54 \%$ in $\mathrm{Tj} 2$, compared to Tj0. In the adults the identification was $68.44 \%$ in Ta1 and $119.58 \%$ in Ta2, compared to Ta0. The treatments tested didn't differ statistically from the control in all the cases. It is recommended to test the algorithms in other areas and in photographs with better quality.

KEYWORDS: point cloud, inventory automatization, ortomosaic.

\section{INTRODUÇÃO}

A área de processamento de imagens digitais capturadas por veículos aéreos não tripulados (VANT) atualmente se apresenta como uma tecnologia de baixo custo quando comparada a métodos tradicionais de coleta de imagens. Desta maneira, os VANT's mostram-se aplicáveis em múltiplos usos, como monitoramento de grandes áreas cultivadas e de áreas de difícil acesso, identificação de pragas e doenças em culturas, detecção de incêndios, entre outros (TAHAR, 2012).

Nessa perspectiva de facilidade de obtenção de informações e, consequentemente, aumento de eficiência e automação dos processos tem-se utilizado VANT's como ferramenta na silvicultura de precisão. Os VANT's vêm sendo empregados, por exemplo, no monitoramento de falhas/sobrevivência em plantios florestais, onde a contagem automática de indivíduos tem-se mostrado como objeto de interesse (CHAVES et al., 2015). Com a crescente inovação na área de técnicas computacionais aliadas à disponibilidade das imagens áreas, diversas pesquisas vêm sendo desenvolvidas, nos últimos anos, acerca da aplicação de métodos para contagem individual de árvores com identificação de padrões em imagens aéreas (GONÇALVES et al., 2016).

A detecção automática de indivíduos arbóreos é de fundamental importância na área florestal, uma vez que possibilita a obtenção de variáveis biométricas (ex. diâmetro e altura das árvores), mostrando-se uma técnica que subsidia a execução de inventários florestais (GIONGO et al., 2010). Além disso, representa uma etapa de amostragem aplicada a inventários florestais, tornando-a cada vez mais eficiente, uma vez que esta é uma etapa onerosa e que demanda tempo (SILVA et al., 2017).

Neste sentido pode-se exemplificar alguns estudos na área florestal nos quais algoritmos para detecção automática de árvores foram desenvolvidos e aplicados. No estudo de Silva et al. (2017), foi empregado o algoritmo de locais máximos implementado no pacote rLiDAR, e o estudo de Falkowski et al. (2014), foi avaliado o desempenho dos algoritmos spacial wavelet analysis (SWA) e variable window filters (VWF) de detecção automatizada de árvores. Ademais, no estudo de 
Frizzarini e Lauretto (2013), o algoritmo DDTB também foi aplicado para a detecção de árvores.

Considerando o exposto, no presente trabalho avaliou-se o desempenho e eficácia dos algoritmos LIDAR 360 e TreeDetect para distinção e contagem automática de árvores jovens e adultas em plantios de Eucalyptus spp.

\section{MATERIAL E MÉTODOS}

\section{Local de Estudo}

A área de estudo consistiu de seis talhões de plantios de Eucalyptus sp. pertencente a empresa Klabin S.A., sendo três talhões de plantios jovens com o híbrido de Eucalyptus grandis x Eucalyptus urophylla com área de 0,72 ha, 0,13 ha e 0,16 ha respectivamente para os talhões 1,2 e 3 . Ademais, foram avaliados três talhões de plantios adultos com Eucalyptus spp. com área de 0,58 ha, 0,24 ha e 0,13 ha respectivamente para os talhões 1,2 e 3 . Os plantios foram estabelecidos com espaçamento de 3,75 x 2,4 m, com densidade de aproximadamente 1.111 mudas/hectare. A idade aproximada do plantio jovem é de 18 meses e do plantio adulto, sete anos.

A área de estudo situa-se no município de Telêmaco Borba-PR. De acordo sistema de classificação climática de Köppen, o município se caracteriza como tipo climático Cfa, subtropical sem estação seca definida, com temperatura no mês mais frio variando de $-3{ }^{\circ} \mathrm{C}$ a $18^{\circ} \mathrm{C}$, temperatura no mês mais quente superior a $22^{\circ} \mathrm{C}$ e precipitação mensal superior a $40 \mathrm{~mm}$ (ALVARES et al., 2014).

\section{Coleta e pré-processamento dos dados}

As fotografias aéreas foram obtidas com o VANT eBee-Ag (Sensefly) equipado com câmera RGB Canon $\mathrm{S} 110 \mathrm{com} 12 \mathrm{Mb}$ de resolução, sensor de 7,44 x $5,58 \mathrm{~mm}$ e distância focal de $4,37 \mathrm{~mm}$ (SENSEFLY, 2018). Foram tomadas, ao total, 20 fotografias aéreas, em setembro de 2015, para recobrimento total dos talhões, com distinção dos plantios jovens e adultos.

\section{Conversão de dados VANT em nuvem de pontos e ortomosaico}

O processamento dos dados VANT foi realizado com o software Agisoft PhotoScan Professional. Para o alinhamento das fotos e formação da nuvem de pontos esparsa utilizou-se como parâmetros gerais a precisão highest com préseleção de referência. Nos parâmetros avançados não foram selecionados o limite de ponto chave e o limite de ponto de ligação, sendo que a opção de ajustar os parâmetros da câmera foram mantidos. Para a geração da nuvem de pontos densa utilizou-se como parâmetros gerais a qualidade highest. Nos parâmetros avançados utilizou-se filtragem de profundidade média e cálculo das cores dos pontos. $\mathrm{Na}$ elaboração do ortomosaico a projeção utilizada foi WGS-84, fuso 22 Sul. O ortomosaico foi utilizado para delimitar a área de interesse e para o recorte da nuvem de pontos referente às mesmas áreas.

\section{Detecção e contagem dos alvos}

Foram testados dois tratamentos, sendo: a) processamento com algoritmo do software LIDAR 360 (GREENVALLEY INTERNATIONAL, 2018) para plantios jovens (Tj1) e plantios adultos (Ta1); e b) processamento no software ArcGis com a ferramenta TreeDetect (HENTZ et al., 2018), também para plantios jovens (Tj2) e plantios adultos (Ta2). A determinação da qualidade do processamento de cada 
tratamento se deu com a comparação dos resultados com a contagem manual em tela, sendo Tj0 o tratamento testemunha plantios jovens e $\mathrm{TaO}$ o tratamento testemunha plantios adultos.

\section{LIDAR360}

Para o processamento no software utilizou-se como entrada dados da nuvem de pontos em formato ".LAS" gerada a partir das imagens coletadas por VANT sendo que ao todo foram realizadas cinco etapas para a detecção dos alvos, sendo: classificação de ground point, criação de digital elevation model (DEM), criação de digital surface model (DSM), criação de canopy height model (CHM) e CHM segmentation. Nesse caso, o algoritmo de detecção de árvores, construído no ambiente do software LIDAR360, foi utilizado.

Para a classificação de ground point os parâmetros de max building size, iteration angle, max terrain angle e iteration distance foram utilizados os valores respectivos de 15, 25, 25 e 1. Na criação do DEM e DSM estabeleceu-se o valor de $0,5 \mathrm{~m}$ para ambos os parâmetros de $\mathrm{X}$ size e $\mathrm{Y}$ size, utilizando o método de interpolação TIN. Os dois modelos foram usados como entrada para criação do $\mathrm{CHM}$, a partir da diferença entre os valores de elevação do DSM em relação ao DTM. Para a segmentação do CHM estabeleceram-se valores de 0,5 m, $6 \mathrm{~m}$ e 50 pixels para as variáveis altura mínima das árvores, altura máxima da árvore e tamanho do buffer para plantios jovens e $5 \mathrm{~m}, 15 \mathrm{~m}$ e 50 pixels para as mesmas variáveis nos talhões adultos.

\section{TreeDetect}

Para o processamento dos dados empregou-se como entrada o ortomosaico em formato tif gerado a partir das imagens coletadas pelo VANT. Para tanto foram criados raster das áreas com plantios jovens e adultos pela intersecção com os shapes de interesse. Nesse processamento, utilizou-se a ferramenta TreeDetect, que consiste numa ferramenta, em linguagem Python, construída em ambiente ArcGis (HENTZ et al., 2018). Os parâmetros adotados para a detecção das árvores foram:

- Valor de conversão: parâmetro obrigatório, podendo variar de 1 a -1. Se o valor de -1 for escolhido, inverte a estrutura da grade.

- Tamanho mínimo: mínima área que uma copa de árvore pode ter, considerando seu menor tamanho possível.

- Taxa de suavização: seleciona-se como padrão de homogeneização de áreas, este valor deve ser incrementado para florestas com alta heterogeneidade.

Para tanto, nos plantios jovens foram usados como padrão para os parâmetros valor de conversão, tamanho mínimo e taxa de suavização os valores de 1,3 e 2 respectivamente. Para os plantios adultos os valores utilizados foram de -1 , 4 e 2 em ordem.

\section{Análise dos dados}

A comparação dos tratamentos com as testemunhas, para a identificação dos indivíduos e delimitação das respectivas copas dos polígonos, possibilitou avaliar o desempenho dos mesmos de acordo com os seguintes critérios:

a) Indivíduos corretamente identificados no método adotado 
no método

b) Erro de omissão: indivíduos existentes na testemunha e não identificadas

c) Erro de comissão: indivíduos não existentes na testemunha e identificados no método

Para verificar a ocorrência de diferença estatística entre os tratamentos (Tj1, Tj2, Ta1 e Ta2), em relação as testemunhas (Tj0 e Ta0) considerando o processamento no software LiDAR 360 e com a ferramenta TreeDetect, foi aplicado um teste $\mathrm{t}$ pareado, a um nível de significância de $5 \%$.

\section{RESULTADOS E DISCUSSÕES}

No tratamento testemunha, o número de indivíduos contabilizados para os talhões jovens 1, 2 e 3 foram 803, 144 e 175 indivíduos respectivamente, totalizando 1.122 indivíduos. No tratamento Tj1, houve subestimativa do total de indivíduos em todos os talhões, do qual foram identificados ao todo 376 indivíduos, sendo, 309, 6 e 61 indivíduos nos talhões 1, 2 e 3, resultando em uma identificação média de $25,83 \%$ (Tabela 1).

TABELA 1. Porcentagem de indivíduos detectados no tratamento Tj1 (plantios jovens) em relação à testemunha (Tj0).

\begin{tabular}{cccccc}
\hline & $\begin{array}{c}\text { Tj1 } \\
\text { (no árvores) }\end{array}$ & $\begin{array}{c}\text { Tj0 } \\
\text { (no árvores) }\end{array}$ & Tj0 - Tj1 & $\begin{array}{c}\text { Porcentagem de } \\
\text { identificação (\%) }\end{array}$ & \multirow{2}{*}{$\boldsymbol{p}^{*}$} \\
\hline Talhão jovem 1 & 309 & 803 & 494 & 38,48 & \multirow{2}{*}{0,180} \\
\cline { 1 - 5 } Talhão jovem 2 & 6 & 144 & 138 & 4,16 & \\
\hline Talhão jovem 3 & 61 & 175 & 114 & 34,85 & \\
\hline Total & 376 & 1.122 & 746 & 25,83 & \\
\hline$a=0,05$ & & & & &
\end{tabular}

No tratamento Tj2 as médias de detecção dos indivíduos foram de 104,54\%, superestimando o resultado em relação a Tj0 em todos os talhões (Tabela 2), sendo que foram identificados para os talhões 1, 2 e 3 respectivamente 829, 154 e 181 indivíduos, totalizando 1.164 indivíduos. Comparativamente, a média de identificação de Tj1 este se manteve acima, mostrando resultados mais próximos do real.

Estatisticamente, as médias dos tratamentos Tj1 e Tj2 não diferiram da do tratamento testemunha, uma vez que os valores de $p$ foram superiores a 0,05 .

TABELA 2. Porcentagem de indivíduos detectados no tratamento Tj2 (plantios jovens) em relação à testemunha (TjO).

\begin{tabular}{|c|c|c|c|c|c|}
\hline & $\begin{array}{c}\mathrm{Tj2} \\
\text { (no árvores) }\end{array}$ & $\begin{array}{c}\text { Tj0 } \\
\text { (no árvores) }\end{array}$ & $\mathrm{Tj} 0-\mathrm{Tj} 2$ & $\begin{array}{l}\text { Porcentagem de } \\
\text { identificação (\%) }\end{array}$ & $p^{*}$ \\
\hline Talhão jovem 1 & 829 & 803 & -26 & 103,24 & \multirow{4}{*}{0,149} \\
\hline Talhão jovem 2 & 154 & 144 & -10 & 106,94 & \\
\hline Talhão jovem 3 & 181 & 175 & -6 & 103,43 & \\
\hline Total & 1.164 & 1.122 & -42 & 104,54 & \\
\hline
\end{tabular}

Nos plantios adultos, em Ta1 observou-se a mesma tendência de subestimar o número total das mudas identificadas em relação a $\mathrm{Ta0}$, do qual identificou-se 516, 196 e 76 mudas nos talhões 1, 2 e 3 respectivamente, totalizando 
788 indivíduos. A média de identificação foi de $68,44 \%$, mantendo-se consideravelmente acima dos resultados médios alcançados pelo mesmo tratamento em plantios jovens (Tabela 3).

TABELA 3. Porcentagem de indivíduos detectados no tratamento Ta1 (plantios adultos) em relação à testemunha ( $\mathrm{Ta} 0)$.

\begin{tabular}{cccccc}
\hline & $\begin{array}{c}\text { Ta1 } \\
\text { (no árvores) }\end{array}$ & $\begin{array}{c}\text { Ta0 } \\
\text { (no árvores) }\end{array}$ & Ta0 - Ta1 & $\begin{array}{c}\text { Porcentagem de } \\
\text { identificação (\%) }\end{array}$ & \multirow{2}{*}{$\boldsymbol{p}^{\text {* }}$} \\
\hline Talhão adulto 1 & 516 & 649 & 133 & 79,51 & \\
\cline { 1 - 5 } Talhão adulto 2 & 196 & 262 & 66 & 74,81 & \multirow{2}{*}{0,051} \\
\cline { 1 - 5 } Talhão adulto 3 & 76 & 149 & 73 & 51,01 & \\
\cline { 1 - 5 } Total & 788 & 1.060 & 544 & 68,44 & \\
\hline
\end{tabular}

${ }^{*} a=0,05$

No tratamento Ta2, a média de detecção das árvores adultas foi de $119,58 \%$, com identificação de 759, 317 e 180 indivíduos respectivamente para os talhões 1, 2 e 3, com maior porcentagem de erro de identificação quando comparado ao mesmo método empregado em plantios jovens. Houve, ainda, a superestimava dos resultados em relação às testemunhas, similarmente aos resultados obtidos em Tj2. Os resultados de Ta1 foram próximos aos resultados computados em Ta0 (Tabela 4).

As médias dos tratamentos Ta1 e Ta2 também não diferiram estatisticamente do tratamento testemunha. Portanto, estatisticamente, tanto para o plantio jovem quanto para o plantio adulto, não houve diferença da aplicação de diferentes algoritmos com relação às testemunhas.

TABELA 4. Porcentagem de indivíduos detectados no tratamento Ta2 (plantios adultos) em relação à testemunha ( $\mathrm{Ta} 0)$.

\begin{tabular}{cccccc}
\hline & $\begin{array}{c}\text { Ta2 } \\
\text { (no árvores) }\end{array}$ & $\begin{array}{c}\text { Ta0 } \\
\text { (no árvores) }\end{array}$ & $\begin{array}{c}\text { Ta0 - Ta2 } \\
\text { (no árvores) }\end{array}$ & $\begin{array}{c}\text { Porcentagem de } \\
\text { identificação (\%) }\end{array}$ & $\boldsymbol{p}^{*}$ \\
\hline Talhão adulto 1 & 759 & 649 & -110 & 116,95 & \multirow{2}{*}{0,108} \\
\cline { 1 - 4 } Talhão adulto 2 & 317 & 262 & -55 & 120,99 & 120,81 \\
\hline Talhão adulto 3 & 180 & 149 & -31 & 119,58 & \\
\hline Total & 1.256 & 1.060 & -196 &
\end{tabular}

No trabalho de Buck et al. (2017), no qual utilizou-se laser terrestre e aplicação do algoritmo Tree Detection in Plantation em povoamentos de Eucalyptus spp., resultou na detecção de $95 \%$ em plantios de cinco anos e $96 \%$ de identificação em plantios de quatro a dois anos. Os valores encontrados para tratamentos $\mathrm{Tj} 1$, Ta1 e Ta2 nesse trabalho foram inferiores aos encontrados no estudo supracitado, sendo que o tratamento que mais se aproximou foi Tj2, porém considerando um valor superestimado. Os resultados obtidos em Ta1, Tj2 e Ta2 foram próximos aos encontrados por Kankare et al. (2015), com precisão global de detecção de $71,2 \%$, e por Olofsson et al. (2014), com precisão de $87 \%$. O único resultado discrepante dos autores foi Tj1, no qual valores inferiores de precisão foram detectados.

$\mathrm{Na}$ pesquisa de Hentz et al. (2018) os resultados de detecção com o TreeDetect superestimaram a contagem em tela, do qual foi encontrado o valor máximo de 4,69\% de erro de detecção em plantios jovens de Eucalyptus spp. e ENCICLOPÉDIA BIOSFERA, Centro Científico Conhecer - Goiânia, v.15 n.28; p.369 2018 
média de $2,75 \%$ de erro nos mesmos plantios. Comparativamente ao estudo, o resultado de Hentz el al. (2018) se aproxima com os encontrados em Tj2, em que houve a superestimação média do total de individuos de $4,54 \%$. Em relação a Tj1 os resultados se distanciaram, visto que houve a subestimação média dos resultados em $74,17 \%$.

Foram calculados, para todos os tratamentos (jovens e adultos), erros de omissão (indivíduos existentes na testemunha e não identificadas no método) e de comissão (indivíduos não existentes na testemunha e identificados no método). $\mathrm{Na}$ Tabela 5 pode-se observar o total de indivíduos computados em ambos os erros nos tratamentos Tj1 e Tj2.

TABELA 5. Quantificação dos erros obtidos nos tratamentos em plantios jovens (Tj1 e Tj2).

\begin{tabular}{cccc}
\hline Tratamento & Talhão & $\begin{array}{c}\text { erro de omissão } \\
\text { (indivíduos) }\end{array}$ & $\begin{array}{c}\text { erro de comissão } \\
\text { (indivíduos) }\end{array}$ \\
\hline \multirow{2}{*}{ Tj1 } & 1 & 565 & 71 \\
& 2 & 140 & 2 \\
\hline \multirow{2}{*}{ Tj2 } & 3 & 123 & 9 \\
\hline & 1 & 11 & 37 \\
\hline & 2 & 2 & 12 \\
\hline
\end{tabular}

Em Tj1 observou-se maior quantidade de erros quando comparado à Tj2. Os erros de omissão médio, em relação ao número total de mudas (Tj0), foram de $79,29 \%$ em Tj1 e de 1,11\% em Tj2, sendo esta diferença evidenciada em função de Tj1 ter subestimado o número total de mudas e Tj2 ter superestimado. Quanto ao erro de comissão médio em relação a Tj0 foi de $5,12 \%$ para Tj1 e de $5,65 \%$ para $\mathrm{Tj} 2$, sendo que Tj2 apresentou maior erro pelo fato desse tambem de superestimar o número total de mudas. Ainda, comparou-se o erro de comissão médio em relação ao total de indivíduos identificados, que foi de 23,69\% para Tj1 e de 5,37\% para Tj2, evidenciando que o total de mudas identificadas por ambos os métodos foram identificadas erroneamente. Nos tratamentos Ta1 e Ta2 também foram obervados os mesmo erros obtidos em Tj1 e Tj2 (Tabela 6).

TABELA 6. Quantificação dos erros obtidos nos tratamentos em plantios adultos (Ta1 e Ta2).

\begin{tabular}{cccc}
\hline Tratamento & Talhão & $\begin{array}{c}\text { erro de omissão } \\
\text { (indivíduos) }\end{array}$ & $\begin{array}{c}\text { erro de comissão } \\
\text { (indivíduos) }\end{array}$ \\
\hline \multirow{3}{*}{ Ta1 } & 1 & 257 & 124 \\
& 2 & 117 & 51 \\
\hline \multirow{2}{*}{ Ta2 } & 3 & 86 & 13 \\
\hline & 1 & 8 & 118 \\
\hline & 2 & 3 & 58 \\
\hline
\end{tabular}

Nos plantios adultos, em Ta1 foram observados erros médio de omissão superiores aos encontrados em Ta2. A média de erro de omissão em T1a foi de 
$47,32 \%$ e de $2,13 \%$ em Ta2, em relação à Ta0. No erro de comissão em relação à $\mathrm{Ta} 0$, as médias em Ta1 e em Ta2 foram, respectivamente, 15,77\% e 21,72\%. Já com relação ao erro de comissão das mudas identificadas por cada método, a média foi de $22,39 \%$ em Ta1 e de 18,13\% em Ta2, evidenciando uma identificação equivocada de mudas em ambos os métodos. Os erros de comissão, no caso dos plantios adultos, podem ser justificados pelo adensamento das árvores, maior que em plantios jovens, pois dessa forma as identificações de galhos laterais como árvores contribuíram para o aumento desse tipo de erro.

Na Figura 1 pode-se visualizar a distribuição de marcação de copas em Tj1, Ta1, Tj2 e Ta2. Em Ta1 houve uma homogeneização da marcação de copas, com não marcação em maior parte nas bordas das imagens. Em Tj1 houve a marcação excessiva em algumas regiões da imagem enquanto que em outras regiões, como por exemplo, a região central, que não houve a marcação de praticamente nenhuma copa, sendo como um todo classificado como uma marcação não homogênea. No Tj2 e Ta2 a marcação das copas se mostrou mais homogênea, não se comprometendo com a não marcação das regiões de borda.
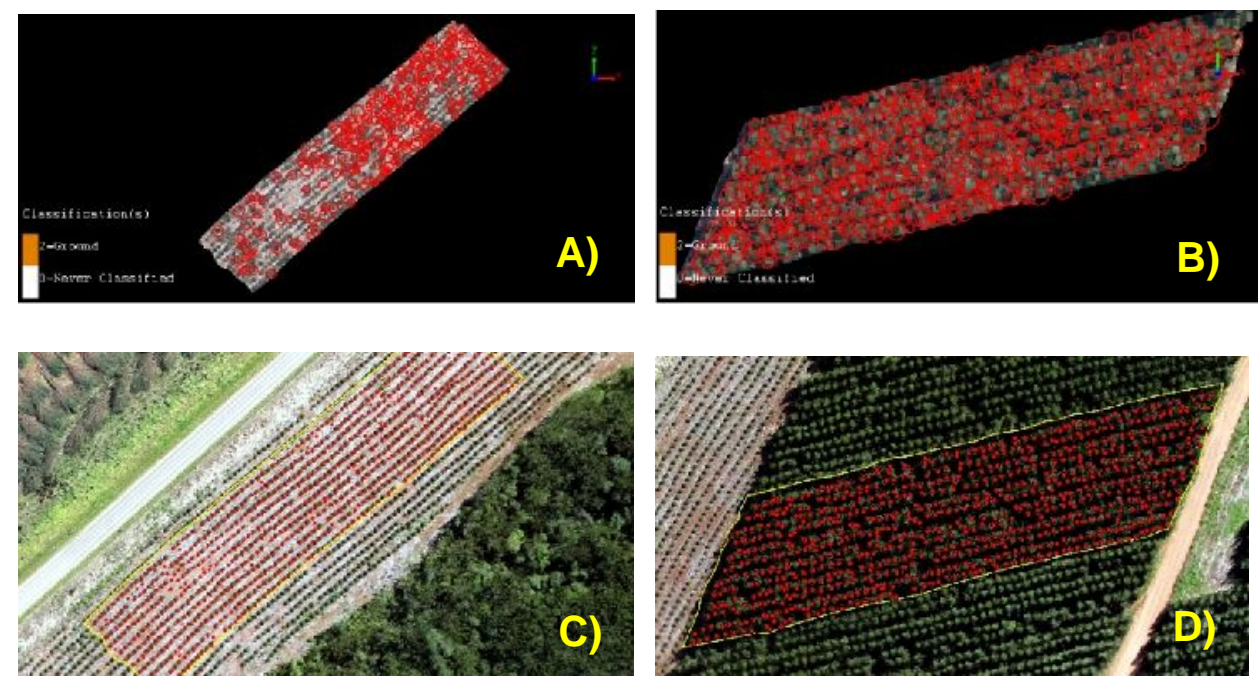

FIGURA 1. Demarcação das copas nos plantios jovens e adultos pelo software LiDAR 360 e pelo Tree Detect. a) tratamento Tj1. b) tratamento Ta1. c) tratamento Tj2. d) tratamento Ta2.

Nos dois tratamentos com o software LiDAR (Tj1 e Ta1) foram observados erros de omissão maiores que de comissão, influindo em subestimativa dos resultados, sendo que esses efeitos ainda foram ressaltados nas áreas de borda (Figura 2). Um ponto importante foi que o modelo não teve boa distinção dos pontos do solo, fato este que influenciou respectivamente na segmentação dos objetos de interesse e, também, na contagem dos objetos como em Tj1, que a classificação dos pontos do chao se manteve abaixo do esperado, sendo que não houve a distinção visual das mudas no modelo. Nos plantios adultos o número de pontos que caracterizaram o terreno não foram suficientes para gerar o modelo digital do terreno. 

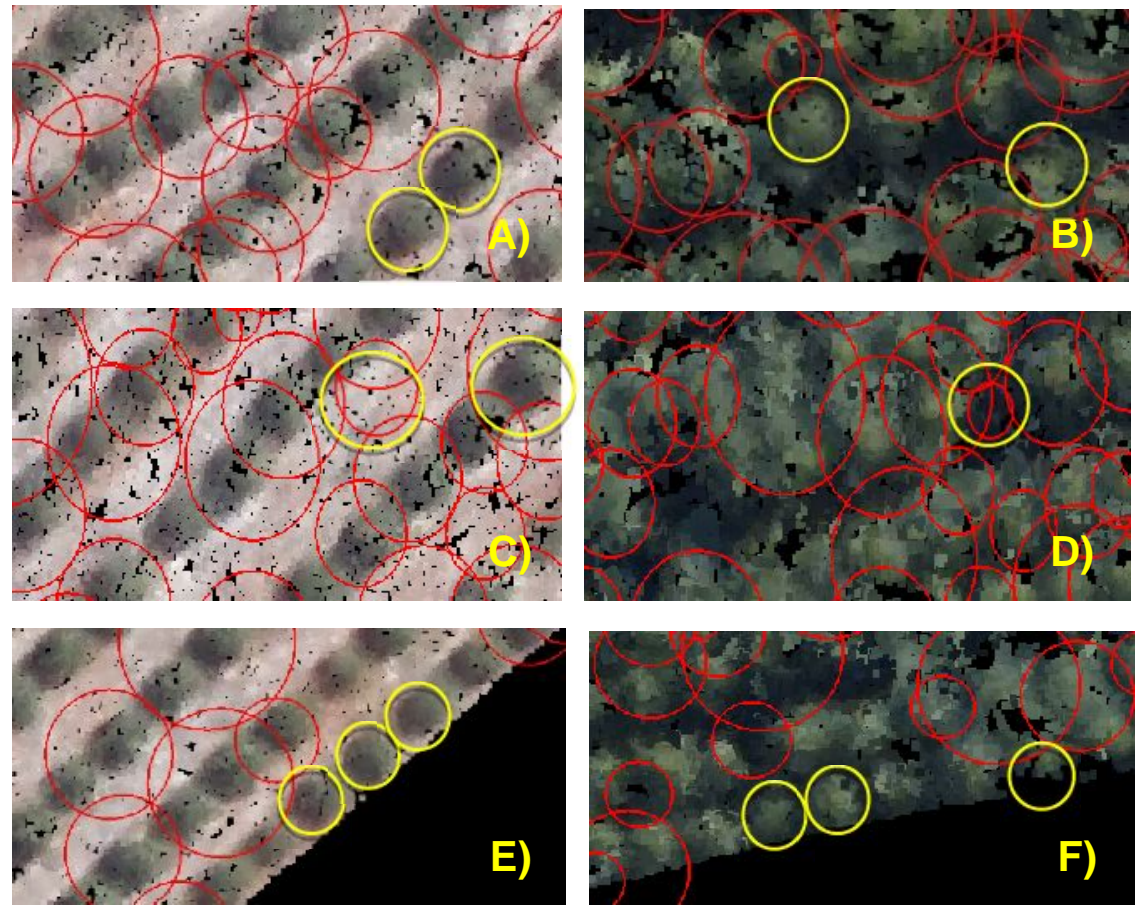

FIGURA 2. Erros identificados na demarcação dos indivíduos pelo LiDAR 360, destacados em amarelo. a) indivíduos contidos na nuvem e não marcados em Tj1. b) indivíduos contidos na nuvem e não marcados em Ta1. c) indivíduos não existentes na nuvem e marcados em Tj1. d) indivíduos não existentes na nuvem e marcados em Ta1. e) efeito borda em Tj1. f) efeito borda em Ta1.

Nos tratamentos com a ferramenta TreeDetect (Tj2 e Ta2) observou-se uma maior quantidade de erros de comissão do que de omissão, resultando em superestimativa dos resultados. Os efeitos de borda não foram observados nesses tratamentos (Figura 3).
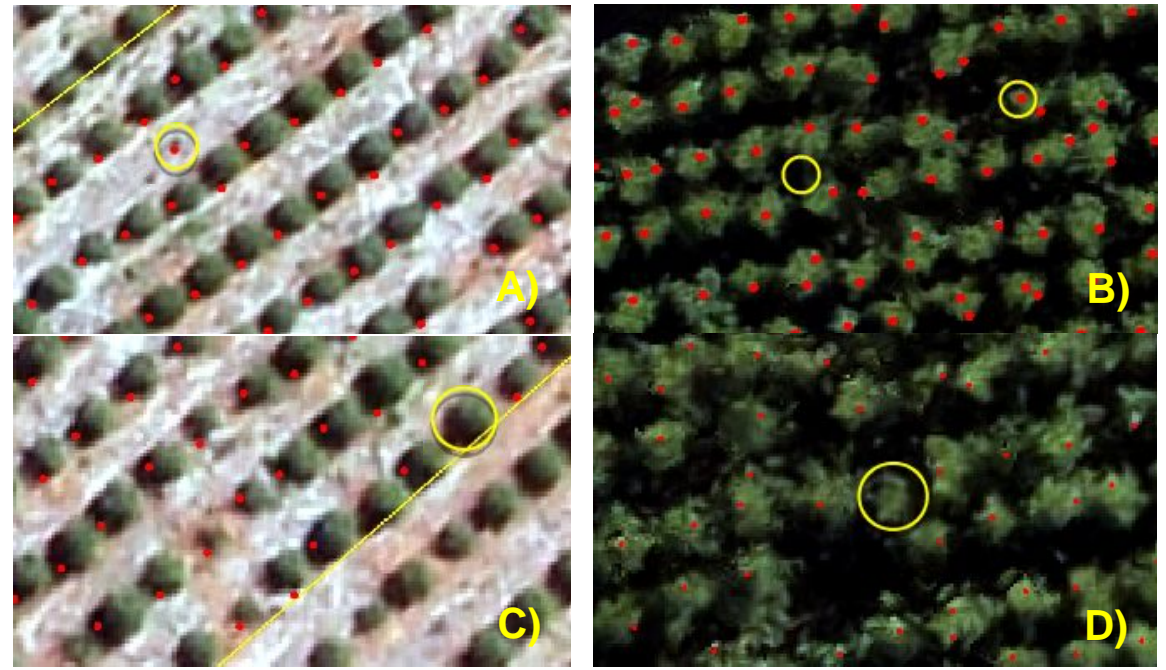

FIGURA 3. Erros identificados na demarcação dos indivíduos pelo TreeDetect, destacados em amarelo. a) indivíduos não existentes na nuvem e marcados em Tj2. b) indivíduos não existentes na nuvem e marcados em Ta2. c) indivíduos contidos na nuvem e não marcados em Tj2. d) indivíduos contidos na nuvem e não marcados em Ta2. 
Uma forma de corrigir esses erros principalmente para os tratamentos $\mathrm{Tj} 1 \mathrm{e}$ Ta1 seria a realização de vôos mais baixos e/ou com câmeras de maior resolução. $O$ maior recobrimento lateral e longitudinal bem como um maior recobrimento do entorno da área de interesse para elaboração dos ortomosaicos e respectivamente das nuvens de pontos tambem são pontos importantes que podem ser melhorados. $O$ auxilio de pontos de controle para obtenção das fotos ainda pode ocasionar melhorias quanto a classificação e contagem dos indivíduos, uma vez que neste caso não foram utilizados.

\section{CONCLUSÕES}

Nos plantios jovens os melhores resultados visuais foram obtidos em Tj2, uma vez que em Tj1 houve uma expressiva subestimativa do número total de indivíduos contabilizados em Tj0.

Nos plantios adultos (Ta1 e Ta2) os resultados de marcação de copas foram visualmente mais homogêneos em relação a Ta0, porém Ta2 ainda se manteve com uma melhor classificação.

Nos tratamentos com o software LiDAR 360 houve subestimativa dos resultados, influenciado pela qualidade da nuvem de pontos e pelo efeito de borda onde não houve a identificação dos indivíduos. Nos tratamentos com a ferramenta TreeDetect houve a superestimativa dos resultados.

Percebeu-se que as áreas com maiores erros de detecção observados apresentavam qualidade precária da imagem, o que dificultou a visualização das árvores. Portanto, recomenda-se testar os mesmos algoritmos em outras áreas e em fotografias com melhor qualidade com o intuito de aumentar a acuidade dos resultados produzidos.

\section{AGRADECIMENTOS}

Os autores agradecem ao CNPq (Conselho Nacional de Desenvolvimento Científico e Tecnológico) pela concessão das bolsas de estudos (iniciação científica e pós-graduação). Agradecem a empresa Klabin, especialmente os Engenheiros Florestais Emerson Roberto Schoeninger e Yuri Accioly que forneceram dados para a realização desse presente trabalho.

\section{REFERÊNCIAS}

ALVARES, C. A.; STAPE, J. L.; SENTELHAS, P. C.; GONÇALVES, J. L. M.; SPAROVEK, G. Köppen's climate classification map for brazil. Meteorologische Zeitschrift, v. 22, n. 6, p. 711-728, 2014. Disponível em: <http://dx.doi.org/10.1127/0941-2948/2013/0507>. doi: 10.1127/09412948/2013/0507.

BUCK, A. L. B.; LINGNAU, C.; MACHADO, A. M. L.; NETTO, S. P. Detecção de árvores em nuvem de pontos de varredura laser terrestre. Boletim de Ciências Geodésicas, Curitiba, v. 23, n. 1, p. 21-38, 2017. Disponível em: <http://dx.doi.org/10.1590/s198221702017000100002>.

doi: 10.1590/s198221702017000100002.

ChAVES, A. A.; LA SCALEA, R. A.; COltuRATO, A. B.; KAWABATA, C. L. O.; FURTADO, E. L.; BRANCO, K. R. L. J. C. Uso de VANTs e processamento digital de imagens para a quantificação de áreas de solo e de vegetação. Anais XVII 
Simpósio brasileiro de sensoriamento remoto, - SBSR, João Pessoa-PB, Brasil, p. 5642-5649, 2015.2 Disponível em: < http://www.dsr.inpe.br/sbsr2015/files/p1143.pdf>.

FALKOWSKI, M. J.; SMITH, A. M. S.; GESSLER, P. E.; HUDAK, A.T.; VIERLING, L. A.; et al. The influence of coniferous forest canopy cover on the accuracy of two individual tree measurement algorithms using LiDAR data. Canadian Journal of Remote Sensing, v. 34, n. 2, p. 338-350, 2014. Disponível em: < https://www.tandfonline.com/doi/abs/10.5589/m08-055>. doi: 10.5589/m08-055.

FRIZZARINI, C.; LAURETTO, M.S. Proposta de um algoritmo para indução de arvores de classificação para dados desbalanceados. Escola de Artes, Ciências e Humanidades - Universidade de São Paulo (EACH-USP). São Paulo, p. 722-733, 2013. Disponível em: < http://www.lbd.dcc.ufmg.br/colecoes/sbsi/2013/0065.pdf>.

GIONGO, M.; KOEHLER, H.S.; MACHADO, S.A.; KIRCHNER, F.F.; MARCHETTI, M. LIDAR: princípios e aplicações florestais. Pesquisa Florestal Brasileira, Colombo, v. 30, n. 63, p. 231-244, ago-out, 2010. Disponível em: <http://dx.doi.org/10.4336/2010.pfb.30.63.231 >. doi: 10.4336/2010.pfb.30.63.231.

GONÇALVES, W. G.; RIBEIRO, H. M. C.; MORALES, G.P.; FILHO, H.R.F.; ALMEIDA, A.C. Classificação de estratos florestais utilizando redes neurais artificiais e dados de sensoriamento remoto. Revista Ambiente \& Água, Taubaté, vol. 11, n. 3, p. 612-624, jul-set de 2016. Disponível em: <http://dx.doi.org/10.4136/ambiagua.1871>. doi: 10.4136/ambi-agua. 1871 .

GREENVALLEY INTERNATIONAL. LiDAR360. Version 2.1, 2018. Disponível em:< https://greenvalleyintl.com/software/software-downloads/>. Acessado em: 16/08/2018.

HentZ, A. M. K.; CORTE, A. P. D.; PÉLliCO NeTO, S.; STRAGER, M. P.; SCHOENINGER, E.R. Treedetection: automatic tree detection using UAV-based data. Revista Floresta, Curitiba-PR, v. 48, n. 3, p. 393-402, jul-set, 2018. Disponível em: <http://dx.doi.org/10.5380/rf.v48i3.56150>. doi: 10.5380/rf.v48i3.56150.

KANKARE, V.; LIANG, X.; VASTARANTA, M.; YU, X.; HOLOPAINEN, M.; HYYPPA, $J$. Diameter distribution estimation with laser scanning based multisource single tree inventory. ISPRS Journal of Photogrammetry and Remote Sensing, v. 108, p. 161-171, 2015. Disponível em: <http://dx.doi.org/10.1016/j.isprsjprs.2015.07.007>. doi: 10.1016/j.isprsjprs.2015.07.007.

OLOFSSON, K.; HOLMGREN, J.; OLSSON, H. Tree Stem and Height Measurements using Terrestrial Laser Scanning and the RANSAC Algorithm. Remote Sensing. v. $6, \quad p$ 4323-4344, 2014. Disponível em: <http://dx.doi.org/10.3390/rs6054323>. doi: 10.3390/rs6054323.

SENSEFLY. eBee The professional mapping drone. 2018. Disponível em: < https://www.sensefly.com/drone/ebee-mapping-drone/>. Acessado em:15/07/2018.

SILVA, I.L.G.; SILVA, C.A.; KLAUBERG, C.; MELLO, J.M. Detecção de árvores individuais em área florestal mista de coníferas por meio de dados LiDAR ENCICLOPÉDIA BIOSFERA, Centro Científico Conhecer - Goiânia, v.15 n.28; p. $374 \quad 2018$ 
aerotransportando. Advances in Forestry Science, Cuiabá, v. 4, n. 2, p. 107-112, $2017 . \quad$ Disponível

em:

http://periodicoscientificos.ufmt.br/ojs/index.php/afor/article/view/4067/pdf>.

TAHAR, K. N. A new approach on slope data acquisition using unmanned aerial vehicle. International Journal of Research and Reviews in Applied Sciences, Pakistan, v. 13, p. 780-785, 2012. Disponível em: <http://dx.doi.org/10.5897/IJPS12.142>. doi: 10.5897/IJPS12.142. 